Ann. Génét. Sél. anim., I978, 10 (4), 533-540.

\title{
Coefficient de parenté et dispersion en population finie. Incidence de la mutation
}

\author{
Gérard GÉRY \\ U.E.R. Mathématiques, Université de Lyon $I$, \\ Math $I$ cycle \\ 43, boulevard du II-Novembre-I9I8, \\ 69621 Villeurbanne, France
}

\section{Résumé}

La biologie moléculaire met en évidence de nombreux polymorphismes. Toute substitution d'un acide aminé à un autre est une mutation qui produit un nouveau gène, un nouvel allèle. La notion de " perte de parenté " traduit ce phénomène. Le coefficient de parenté gamètique $\mathrm{X}(t)$ conditionné par la connaissance de la $n^{\mathrm{e}}$ génération; probabilité d'identité de deux gamètes tirés au hasard avec remise dans le réservoir gamètique de $1 \mathrm{a} n+\mathrm{I}^{\mathrm{e}}$ génération, admet pour espérance à priori le coefficient de parenté $f(t)$ de MALÉcơ. Le but de cet article est l'étude du

rôle de la mutation sur l'évolution de sa variance à priori.
Le coefficient de parenté $f(t)$ converge vers I en absence de mutation et vers $f(\infty) \# \frac{\mathrm{I}}{\mathrm{x}+4 \mathrm{~N} u}$
lorsque le taux de mutation est $u$. lorsque le taux de mutation est $u$

La variance de $\mathrm{X}(t)$ converge vers o en absence de mutation et vers var $(\mathrm{X}(\infty)) \# \frac{2 \mathrm{~N} u}{3}$ $\frac{5+2 \mathrm{~N} u}{(3+4 \mathrm{~N} u)(\mathrm{I}+2 \mathrm{~N} u)(\mathrm{I}+4 \mathrm{~N} u)^{2}}$ lorsque le taux de mutation $u$ est del'ordre de $\frac{\mathrm{I}}{2 \mathrm{~N}}$. La mutation a pour effet d'augmenter la valeur asymptotique de cette variance et, d'augmenter la vitesse de convergence vers cette limite.

\section{Introduction}

Il est intuitivement évident que la limitation de l'effectif d'une population isolée implique des liens d'apparentement entre les individus. Si nous étudions l'évolution d'un locus A (MALÉ́coT, I966) a proposé comme indicateur de la consanguinité attaché à ce locus un " coefficient de consanguinité moyen " (ou coefficient de parenté attaché au locus $\mathrm{A}$ " Probabilité à priori pour que deux gamètes issus de la population dans sa phase haploïde descendent d'un même locus ancêtre commun ".

Pour traduire l'effet de la mutation il a été proposé le concept de " perte de 
parenté " (SERANT, I976) qui généralise 1'hypothèse de béta-mutation introduite par MaLÉCOT, Ig66.

De très nombreux travaux ont été consacrés à ce problème (MALÉcóT, JACQUard, Morton, Kimura, Serant), dans la plupart de ces études c'est une évolution en moyenne qui est envisagée. Le problème de la dispersion de ces apparentements n'a été que rarement abordé (JACQUARD, I975). mène.

Notre but est d'examiner le rôle de la mutation sur l'évolution de ce phéno-

\section{Modèle stochastique}

Nous considérons une population isolée, d'effectif constant, $\mathrm{N}$ diplodes, panmictique, à générations séparées, à sexes non séparés.

Nous utilisons le modèle classique suivant :

$\mathrm{G}(t)$ : réservoir gamètique des $2 \mathrm{~N}$ gamètes utiles de la te génération.

$\mathrm{D}(t): \mathrm{N}$ diploïdes de la $t$ e génération issus de $\mathrm{G}(t)$ par tirage sans remise et exhaustif de $\mathrm{N}$ couples de gamètes.

$\mathrm{R}(t)$ : réservoir infini gamètrique produit par $\mathrm{D}(t)$ après intervention du phénomène de mutation.

$\mathrm{G}(t+\mathrm{I})$ : réservoir gamètique des $2 \mathrm{~N}$ gamètes utiles à la $t+\mathrm{I}^{\mathrm{e}}$ génération.

Pour l'étude du problème haploïde envisagé, il est équivalent de schématiser le modèle en prenant pour loi de passage de $\mathrm{G}(t)$ à $\mathrm{G}(t+\mathrm{I})$ une multinomiale de $2 \mathrm{~N}$ tirages indépendants avec remise.

Nous supposons l'absence de migration et de sélection. Nous notons $u$ le taux de mutation, et nous supposons que chaque mutation fait apparaître une nouvelle forme allèle n'ayant pas existé dans la population avant cette mutation (cette hypothèse abordée par KIMURA et CROW I964 est très satisfaisante en génétique moléculaire).

Nous nous plaçons dans le cas de "perte de parenté " introduite par Serant, si au cours d'une descendance entre un gamète $\Gamma$ de $\mathrm{G}(t)$ et son géniteur $\Gamma^{\prime}$ de $\mathrm{G}(t-\mathrm{I})$ se réalise l'événement " perte de parenté " la probabilité pour que $\Gamma$ porte la forme allèle $A_{k}$, sachant que $\Gamma^{\prime}$ portait la forme $A_{j}$, est égale à la probabilité pour que $\Gamma$ porte $A_{j}$.

Nous dirons que 2 gamètres de $\mathrm{G}(t)$ sont identiques s'ils descendent d'un ancêtre commun sans perte de parenté pour A. Cette relation d'identité est alors analogue à la relation " deux gamètes portent la même forme allèle du gène $A$ ".

Nous notons $\left(x_{1}=n_{1}, \ldots, x_{k}=n_{k}\right)$ les effectifs de la partition en classes d'identité par A du réservoir $\mathrm{G}(t)$.

$$
\sum_{l=1}^{k} n_{1}=2 \mathrm{~N} \quad p_{1}(t)=\frac{n_{1}}{2 \mathrm{~N}}
$$

Nous supposons connue la constitution du réservoir initial $(\mathrm{GO}):\left(p_{i}(\mathrm{O})\right)$. Nous appelons "coefficient de parenté gamétrique conditionné " à la $t_{0}$ génération, la probabilité d'identité de 2 gamètes tirés au hasard dans $\mathrm{G}(t)$, connaissant la constitution de $\mathrm{G}(t)$, avec remise.

$$
\mathrm{X}(t)=\sum_{l=1}^{k} p_{l}^{2}(t)
$$


Le théorème des probabilités totales permet de calculer la probabilité a priori d'identité de 2 gamètes tirés au hasard dans $\mathrm{G}(t)$.

$f(t)=\mathrm{E}(\mathrm{X}(t))=\mathrm{E}\left(\Sigma p^{2}{ }_{1}(t)\right)=\sum_{\left(n_{1}, \ldots, n_{k}\right)}$ Prob $\left(x_{1}=n_{1}, \ldots, x_{k}=n_{k}\right)$ Prob (ident $\left(x_{1}=n_{1}, \ldots\right)$

où $f(t)$, coefficient de parenté gamétique de MALÉCOT, représente une espérance mathématique.

Intéressons-nous à la variance de $\mathrm{X}(t)$ pour étudier la dispersion de ce coefficient de parenté.

$$
\operatorname{Var}(\mathrm{X}(t))=\mathrm{E}\left(\sum_{1} p_{1}^{4}(t)+\sum_{1 \neq m} p^{2}{ }_{1}(t) p^{2}{ }_{m}(t)\right)-f^{2}(t)
$$

\section{Équations de récurrence}

La probabilité d'identité entre 2 gamètes tirés au hasard avec remise dans $\mathrm{G}(t+\mathrm{I})$ est $\mathrm{X}(t+\mathrm{I})$; les 2 gamètes proviennent d'un même gamète de $\mathrm{G}(t)$ avec la probabilité $\frac{\mathrm{I}}{2 \mathrm{~N}}$, et sans mutation avec la probabilité $(\mathrm{I}-u)^{2}$; et de 2 gamètes différents avec la probabilité $\mathrm{I}-\frac{\mathrm{I}}{2 \mathrm{~N}}$, et sans mutation avec la probabilité $(I-u)^{2}$.

soit :

$$
f(t+\mathrm{I})=(\mathrm{I}-u)^{2}\left(\frac{\mathrm{I}}{2 \mathrm{~N}}+\left(\mathrm{I}-\frac{\mathrm{I}}{2 \mathrm{~N}}\right) f(t)\right)
$$

d'où :

$$
\begin{aligned}
f(t)=\frac{\mathrm{I}}{2 \mathrm{~N}} \frac{(\mathrm{I}-u)^{2}}{\mathrm{I}-(\mathrm{I}-u)^{2}\left(\mathrm{I}-\frac{\mathrm{I}}{2 \mathrm{~N}}\right)}+(\mathrm{I}-u)^{2 t}\left(\mathrm{I}-\frac{\mathrm{I}}{2 \mathrm{~N}}\right)^{t} \\
\left(f(0)-\frac{(\mathrm{I}-u)^{2}}{2 \mathrm{~N}\left(\mathrm{I}-(\mathrm{I}-u)^{2}\left(\mathrm{I}-\frac{\mathrm{I}}{2 \mathrm{~N}}\right)\right)}\right.
\end{aligned}
$$

La probabilité d'identité entre 4 gamètes pris au hasard avec remise dans $\mathrm{G}(t+\mathrm{I})$ est $\sum_{\mathbf{1}} p^{4}{ }_{1}(t+\mathrm{I})$

Les 4 gamètes tirés avec remise dans $\mathrm{G}(t+\mathrm{I})$ sont issus de $\mathrm{G}(t)$, sans mutation, selon les situations suivantes, qui s'excluent, avec pour probabilité d'identité (conditionnée par la connaissance de $\mathrm{G}(t)$ ) :

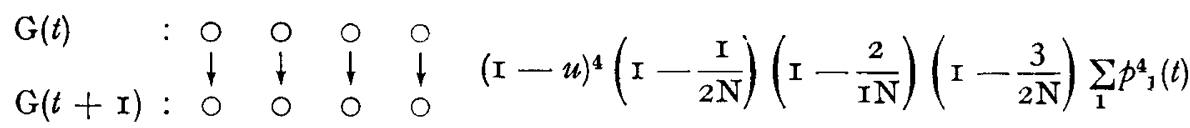

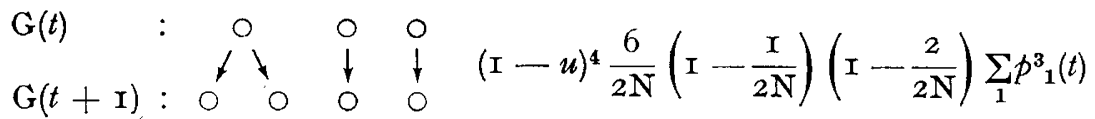




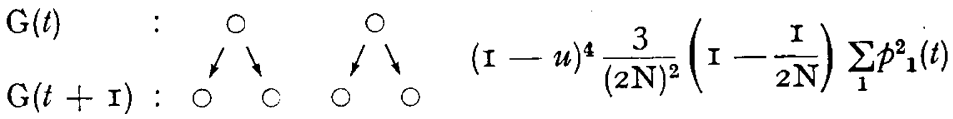

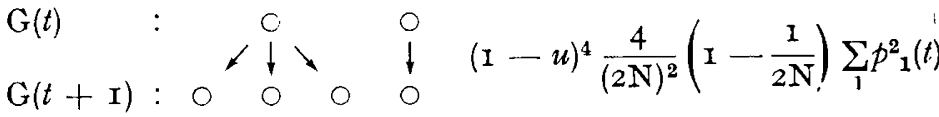

$$
\begin{aligned}
& \mathrm{G}(t): \begin{array}{cccc}
0 \\
\mathrm{G}(t+\mathrm{I}): 0 & 0 & 0 & (\mathrm{I}-u)^{4} \frac{\mathrm{I}}{(2 \mathrm{~N})^{3}}
\end{array}
\end{aligned}
$$

Le théorème des probabilités conditionnées donne :

$$
\begin{aligned}
\mathrm{E}\left(\sum_{1} p_{1}^{4}(t+\mathrm{I})\right) & =\sum_{1} \overline{p_{1}^{4}{ }_{1}(t+\mathrm{I})}=(\mathrm{I}-u)^{4}\left[\left(\mathrm{I}-\frac{\mathrm{I}}{2 \mathrm{~N}}\right)\left(\mathrm{I}-\frac{2}{2 \mathrm{~N}}\right)\left(\mathrm{I}-\frac{3}{2 \mathrm{~N}}\right) \sum_{1} p_{1}^{4}(t)\right. \\
+ & \left.\frac{3}{\mathrm{~N}}\left(\mathrm{I}-\frac{\mathrm{I}}{2 \mathrm{~N}}\right)\left(\mathrm{I}-\frac{2}{2 \mathrm{~N}}\right) \sum_{1} \overline{p^{3}{ }_{1}(t)}+\frac{7}{(2 \mathrm{~N})^{2}}\left(\mathrm{I}-\frac{\mathrm{I}}{2 \mathrm{~N}}\right) \sum_{1} \overline{p^{2}{ }_{1}(t)}+\frac{\mathrm{I}}{(2 \mathrm{~N})^{3}}\right]
\end{aligned}
$$

La probabilité d'identité 2 à 2 de 4 gamètes pris au hasard avec remise dans $\mathrm{G}(t+\mathrm{I})$ est $\sum_{1 \neq m} p_{1}^{2}(t+\mathrm{I}) p^{2}{ }_{m}(t+\mathrm{I})$.

Les 4 gamètes identiques 2 à 2 tirés dans $\mathrm{G}(t+\mathrm{I})$ sont issus de $\mathrm{G}(t)$, sans mutation, selon les situations suivantes, qui s'excluent, avec pour probabilité d'identité (conditionnée par la connaissance de $\mathrm{G}(t)$ ) :

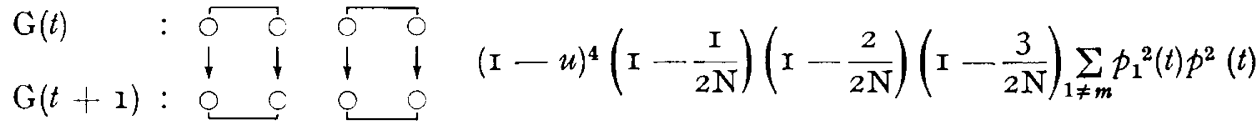

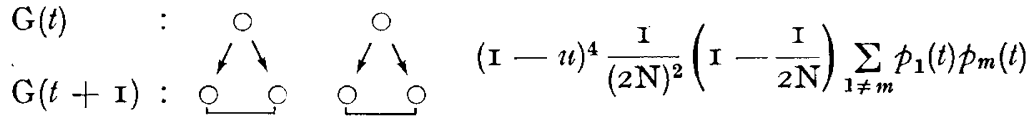

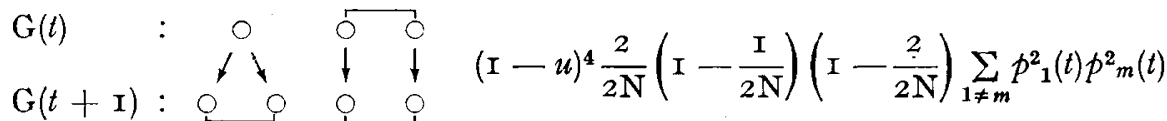

Le théorème des probabilités conditionnées donne :

$$
\begin{array}{r}
\sum_{1 \neq m} \overline{p_{1}^{2}(t+\mathrm{I}) p^{2}{ }_{m}(t+\mathrm{I})}=(\mathrm{I}-u)^{4}\left[\left(\mathrm{I}-\frac{\mathrm{I}}{2 \mathrm{~N}}\right)\left(\mathrm{I}-\frac{2}{2 \mathrm{~N}}\right)\left(\mathrm{I}-\frac{3}{2 \mathrm{~N}}\right) \sum_{1 \neq m} \overline{p^{2}{ }_{1}(t) p^{2}{ }_{m}(t)}\right. \\
\left.+\frac{\mathrm{I}}{(2 \mathrm{~N})^{2}}\left(\mathrm{I}-\frac{\mathrm{I}}{2 \mathrm{~N}}\right) \sum_{1 \neq m} \overline{p_{1}(t) p_{m}(t)}+\frac{2}{2 \mathrm{~N}}\left(\mathrm{I}-\frac{1}{2 \mathrm{~N}}\right)\left(\mathrm{I}-\frac{2}{2 \mathrm{~N}}\right) \sum_{1 \neq m} \overline{p_{1}{ }^{2}(t) p_{m}(t)}\right]
\end{array}
$$

La probabilité d'identité entre 3 gamètes pris au hasard avec remise dans $\mathrm{G}(t+\mathrm{I})$ est $\sum_{1} p_{1}^{3}(t+\mathrm{I})$. 
Les 3 gamètes tirés avec remise dans $\mathrm{G}(t+\mathrm{I})$ sont issus de $\mathrm{G}(t)$, sans mutation, selon les situations suivantes, qui s'excluent, avec pour probabilité d'identité (conditionnée par la connaissance de $\mathrm{G}(t)$ ) :

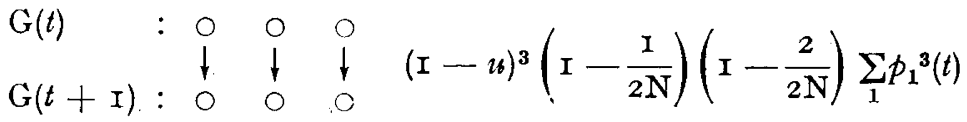

$\mathrm{G}(t) \quad: \quad \mathrm{O} \quad \mathrm{O}$

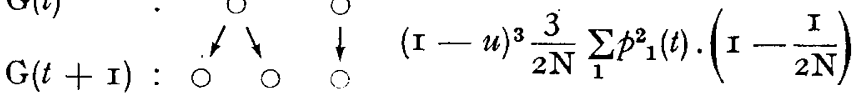

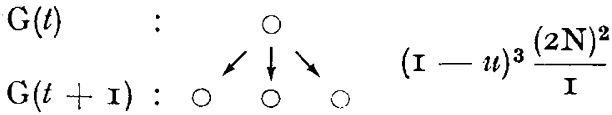

Le théorème des probabilités conditionnés donne :

$$
\begin{aligned}
\sum_{1} \overline{p_{1}^{3}(t+\mathrm{I})}=(\mathrm{I}-u)^{3}\left[\left(\mathrm{I}-\frac{\mathrm{I}}{2 \mathrm{~N}}\right)\left(\mathrm{I}-\frac{2}{2 \mathrm{~N}}\right) \sum_{1} \overline{p^{3}{ }_{1}(t)}\right. \\
+\frac{3}{2 \mathrm{~N}}\left(\mathrm{I}-\frac{\mathrm{I}}{2 \mathrm{~N}}\right) \sum_{1} \overline{p_{1}{ }_{1}(t)}+\frac{\mathrm{I}}{(2 \mathrm{~N})^{2}}
\end{aligned}
$$

Deux gamètes tirés au hasard avec remise dans $\mathrm{G}(t+\mathrm{I})$ sont non identiques, s'ils proviennent sans mutation de 2 classes d'identité distinctes de $\mathrm{G}(t)$, ou si au moins l'un mute.

soit :

$$
\sum_{1 \neq m} \overline{p_{1}(t+\mathrm{I}) p_{m}(t+\mathrm{I})}=(\mathrm{I}-u)^{2}\left(\mathrm{I}-\frac{\mathbf{I}}{2 \mathrm{~N}}\right) \sum_{1 \neq m} \overline{p_{1}(t) p_{m}(t)}+\mathrm{I}-(\mathrm{I}-u)^{2}
$$

La probabilité pour que 3 gamètes tirés avec remise dans $\mathrm{G}(t+\mathrm{I})$, dont 2 sont identiques, est $\sum_{1 \neq m} p_{1}^{2}(t+\mathrm{I}) p_{m}(t+\mathrm{I})$

\begin{tabular}{|c|c|c|c|}
\hline $\begin{array}{l}\mathrm{G}(t) \\
\mathrm{G}(t+\mathrm{I})\end{array}$ & $\begin{array}{rr}0 & 0 \\
1 & 1 \\
0 & 0\end{array}$ & $\begin{array}{l}0 \\
1 \\
0\end{array}$ & $(\mathrm{I}-u)^{3}\left(\mathrm{I}-\frac{\mathrm{I}}{2 \mathrm{~N}}\right)\left(\mathrm{I}-\frac{2}{2 \mathrm{~N}}\right) \sum_{1 \neq m} p_{1}^{2}(t) p_{m}(t)$ \\
\hline $\begin{array}{l}\mathrm{G}(t) \\
\mathrm{G}(t+\mathrm{I})\end{array}$ & $: 0^{\circ}$ & $\begin{array}{l}0 \\
\downarrow \\
0\end{array}$ & $(\mathrm{I}-u)^{3} \frac{\mathrm{I}}{2 \mathrm{~N}}\left(\mathrm{I}-\frac{\mathrm{I}}{2 \mathrm{~N}}\right) \sum_{1 \neq m} p_{1}(t) p_{m}(t)$ \\
\hline $\begin{array}{l}\mathrm{G}(t) \\
\mathrm{G}(t+\mathbf{I})\end{array}$ & $\begin{array}{ll}0 & 0 \\
\downarrow & \downarrow \\
0 & 0\end{array}$ & $\downarrow_{0}^{O}$ mute & $u(\mathrm{I}-u)^{2}\left(\mathrm{I}-\frac{\mathrm{I}}{2 \mathrm{~N}}\right) \sum_{1} p^{2}{ }_{1}(t)$ \\
\hline $\begin{array}{l}\mathrm{G}(t) \\
\mathrm{G}(t+\mathrm{I})\end{array}$ & $: \begin{array}{c}0 \\
11 \\
0\end{array}$ & $f_{0}^{0}$ mute & $u(\mathbf{I}-u)^{2} \frac{\mathbf{I}}{2 \mathrm{~N}}$ \\
\hline
\end{tabular}

Les 3 gamètes sont issus de $\mathrm{G}(t)$ selon les situations suivantes, qui s'excluent, avec pour probabilité (conditionnée par la connaissance de $\mathrm{G}(t)$ ) : 
I e théorème des probabilités conditionnées donne :

$$
\begin{aligned}
& \sum_{1 \neq m} \overline{p_{1}^{2}(t+\mathrm{I}) p_{m}(t+\mathrm{I})}=(\mathrm{I}-u)^{3}\left[\left(\mathrm{I}-\frac{\mathrm{I}}{2 \mathrm{~N}}\right)\left(\mathrm{I}-\frac{2}{2 \mathrm{~N}}\right) \sum_{1 \neq m} \overline{p^{2}(t) p_{m}(t)}\right. \\
& \left.+\frac{\mathrm{I}}{2 \mathrm{~N}}\left(\mathrm{I}-\frac{\mathrm{I}}{2 \mathrm{~N}}\right) \sum_{\mathrm{I} \neq m} \overline{p_{1}(t) p_{m}(t)}\right]+u(\mathrm{I}-u)^{2}\left[\frac{\mathrm{I}}{2 \mathrm{~N}}+\left(\mathrm{I}-\frac{\mathrm{I}}{2 \mathrm{~N}}\right) \sum_{\mathbf{1}} \overline{p^{2}{ }_{1}(t)}\right]
\end{aligned}
$$

Posons : $\alpha=\frac{\mathrm{I}}{2 \mathrm{~N}}$.

La résolution de proche en proche de ces diverses équations nous donne pour résultat :

$$
\begin{aligned}
& \operatorname{Var}(\mathrm{X}(t))=2 u \alpha^{2}(\mathrm{I}-u)^{6}(\mathrm{I}-\alpha)\left[5 \alpha-8 \alpha^{2}+4 \alpha^{3}+u\left(\mathrm{I}-\mathrm{I} 0 \alpha+2 \mathrm{I} \alpha^{2}-\mathrm{I} 4 \alpha^{3}\right)\right. \\
& +u^{2}\left(-2+\mathrm{I} 2 \alpha-25 \alpha^{2}+\mathrm{I} 8 \alpha^{3}\right)+u^{3}\left(\mathrm{I}-7 \alpha+\mathrm{I} 5 \alpha^{2}-\mathrm{I} 0 \alpha^{3}\right) \\
& \left.+u^{4} \alpha\left(\mathrm{I}-3 \alpha+2 \alpha^{2}\right)\right]\left[\mathrm{I}-(\mathrm{I}-u)^{4}(\mathrm{I}-\alpha)(\mathrm{I}-2 \alpha)(\mathrm{I}-3 \alpha]^{-1}\right. \\
& {\left[\mathrm{I}-(\mathrm{I}-u)^{2}(\mathrm{I}-\alpha)\right]^{-2}\left[\mathrm{I}-(\mathrm{I}-u)^{3}(\mathrm{I}-\alpha)(\mathrm{I}-2 \alpha)\right]^{-1}} \\
& +\mathrm{A}(\mathrm{I}-u)^{t}(\mathrm{I}-\alpha)^{t}(\mathrm{I}-2 \alpha)^{t}(\mathrm{I}-3 \alpha)^{t} \\
& +\mathrm{B}(\mathrm{I}-u)^{3 t}(\mathrm{I}-\alpha)^{t}(\mathrm{I}-2 \alpha)^{t} \\
& +\mathrm{C}(\mathrm{I}-u)^{2 t}(\mathrm{I}-\alpha)^{t} \\
& -(\mathrm{I}-u)^{4 t}(\mathrm{I}-\alpha)^{2 t}\left[t_{0}-\frac{\alpha(\mathrm{I}-u)^{2}}{\mathrm{I}-(\mathrm{I}-u)^{2}(\mathrm{I}-\alpha)}\right]^{2}
\end{aligned}
$$

avec :

$$
\begin{aligned}
& \mathrm{C}=2 \alpha(\mathrm{I}-u)^{2}\left(f_{0}-\frac{\alpha(\mathrm{I}-u)^{2}}{\mathrm{I}-(\mathrm{I}-u)^{2}(\mathrm{I}-\alpha}\right) \\
& \left(\frac{u(\mathrm{I}-2 \alpha)+\alpha[3+(\mathrm{I}-u)(5+u-\mathrm{I} \mathrm{I} \alpha)]}{[\mathrm{I}-(\mathrm{I}-u)(\mathrm{I}-2 \alpha)]\left[\mathrm{I}-(\mathrm{I}-u)^{2}(\mathrm{I}-2 \alpha)(\mathrm{I}-3 \alpha)\right]}-\frac{\mathrm{I}}{\mathrm{I}-(\mathrm{I}-u)^{2}(\mathrm{I}-\alpha)}\right) \\
& \mathrm{B}=\frac{4 \alpha(\mathrm{I}-u)}{\mathrm{I}-(\mathrm{I}-u)\left(\mathrm{I}-3^{\alpha}\right)}\left[\sum_{1}{p^{3}}^{3}(0)-\frac{3 \alpha(\mathrm{I}-u)}{\mathrm{I}-(\mathrm{I}-u)(\mathrm{I}-2 \alpha)}\left(f_{0}-\frac{\alpha(\mathrm{I}-u)^{2}}{\mathrm{I}-(\mathrm{I}-u)^{2}(\mathrm{I}-\alpha)}\right.\right. \\
& \left.-\frac{\alpha^{2}(\mathrm{I}-u)^{3}}{\mathrm{I}-(\mathrm{I}-u)^{2}(\mathrm{I}-\alpha)} \cdot \frac{\mathrm{I}+2(\mathrm{I}-u)^{2}(\mathrm{I}-\alpha)}{\mathrm{I}-(\mathrm{I}-u)^{3}(\mathrm{I}-\alpha)(\mathrm{I}-2 \alpha)}\right] \\
& \mathrm{A}=f^{2}{ }_{0}-\left[f_{0}-\frac{\alpha(\mathrm{I}-u)^{2}}{\mathrm{I}-(\mathrm{I}-u)^{2}(\mathrm{I}-\alpha)}\right] \frac{2 \alpha(\mathrm{I}-u)^{2}}{\mathrm{I}-(\mathrm{I}-u)(\mathrm{I}-2 \alpha)} \\
& \frac{u(\mathrm{I}-2 \alpha)+\alpha[3+(\mathrm{I}-u)(5+u-\mathrm{II} \alpha)]}{\mathrm{I}-(\mathrm{I}-u)^{2}(\mathrm{I}-2 \alpha)(\mathrm{I}-3 \alpha)} \\
& -\frac{4 \alpha(\mathrm{I}-u)}{\mathrm{I}-(\mathrm{I}-u)(\mathrm{I}-3 \alpha)}\left[\sum_{1} p^{3}{ }_{1}(\mathrm{o})-\frac{3 \alpha(\mathrm{I}-u)}{\mathrm{I}-(\mathrm{I}-u)(\mathrm{I}-2 \alpha)}\right. \\
& {\left[f_{0}-\frac{\alpha(\mathrm{I}-u)^{2}}{\mathrm{I}-(\mathrm{I}-u)^{2}(\mathrm{I}-\alpha)}\right]} \\
& \left.-\frac{\alpha^{2}(\mathrm{I}-u)^{3}}{\mathrm{I}-(\mathrm{I}-u)^{2}(\mathrm{I}-\alpha)} \cdot \frac{\mathrm{I}+2(\mathrm{I}-u)^{2}(\mathrm{I}-\alpha)}{\mathrm{I}-(\mathrm{I}-u)^{3}(\mathrm{I}-\alpha)(\mathrm{I}-2 \alpha)}\right] \\
& -\frac{\alpha^{2}(\mathrm{I}-u)^{4}}{\left[\mathrm{I}-(\mathrm{I}-u)^{2}(\mathrm{I}-\alpha)\right]\left[\mathrm{I}-(\mathrm{I}-u)^{4}(\mathrm{I}-\alpha)(\mathrm{I}-2 \alpha)(\mathrm{I}-3 \alpha)\right]\left[\mathrm{I}-(\mathrm{I}-u)^{3}(\mathrm{I}-\alpha)(\mathrm{I}-2 \alpha)\right]} \\
& {\left[\mathrm{I}-(\mathrm{I}-u)^{2}(\mathrm{I}-\alpha)[\mathrm{I}-6 \alpha-2 u(\mathrm{I}-2 \alpha)-(\mathrm{I}-u)(\mathrm{I}-2 \alpha)\right.} \\
& \left.\left.\left[\mathrm{I}+4 \alpha-(\mathrm{I}-u)^{2}(\mathrm{I}-\alpha)(\mathrm{I}-6 \alpha)\right]\right]\right]
\end{aligned}
$$




\section{Discussion}

I. - Étude sans mutation $(u=0)$

Nous obtenons :

$$
\begin{aligned}
f(t) & =(\mathrm{I}-\alpha)^{t}\left(f_{0}-\mathrm{I}\right)+\mathrm{I} \\
\operatorname{Var}(\mathrm{X}(t)) & =\mathrm{A}_{0}(\mathrm{I}-\alpha)^{t}(\mathrm{I}-2 \alpha)^{t}\left(\mathrm{I}-3^{\alpha}\right)^{t}+\mathrm{B}_{0}(\mathrm{I}-\alpha)^{t}(\mathrm{I}-2 \alpha)^{t} \\
& +\mathrm{C}_{0}(\mathrm{I}-\alpha)^{t}-(\mathrm{I}-\alpha)^{2 t}\left(f_{0}-\mathrm{I}\right)^{2}
\end{aligned}
$$

avec :

$$
\begin{aligned}
& \mathrm{A}_{0}=f^{2}{ }_{0}+2 f_{0}-\frac{5}{3}-\frac{4}{3} \Sigma p^{3}{ }_{1}(\mathrm{o})+\left(\mathrm{I}-f_{0}\right) \frac{8-\mathrm{II} \alpha}{5-6 \alpha} \\
& \mathrm{B}_{0}=\frac{2}{3}\left(\mathrm{I}-3 f_{0}+2 \Sigma p^{3}{ }_{1}(\mathrm{o})\right) \\
& \mathrm{C}_{0}=\left(\mathrm{I}-f_{0}\right) \frac{2-\alpha}{5-6 \alpha}
\end{aligned}
$$

En l'absence de mutation le coefficient de parenté gamétique moyen $f(t)$ converge asymptotiquement vers $I$ avec la même vitesse de convergence que $(\mathrm{I}-\alpha)^{t}$ vers $\mathrm{o}$.

La variance de ce coefficient de parenté gamétique converge asymptotiquement vers $o$ avec la même vitesse de convergence.

\section{2. - Étude avec mutation $(u \neq 0)$}

Le coefficient de parenté gamétique moyen $f(t)$ converge asymptotiquement vers $f(\infty)(0<f(\infty)<\mathrm{I})$ avec la même vitesse de convergence que $(\mathrm{I}-\alpha)^{t}$ $(\mathrm{I}-u)^{2 t}$ vers 0 . La présence de la mutation a pour rôle de réduire la valeur asymptotique de $f(t)$, et d'augmenter la vitesse de convergence vers cette limite $\left((\mathrm{I}-\alpha)^{t}\right.$ $\left.(\mathrm{I}-u)^{2 t}<(\mathrm{I}-\alpha)^{t}\right)$.

La variance de ce coefficient de parenté gamétique converge asymptotiquement vers une limite non nulle $(\operatorname{Var}(\mathrm{X}(\infty))$ avec la même vitesse de convergence que $f(t)$. La présence de la mutation a pour rôle d'augmenter la valeur asymptotique de cette variance, et d'augmenter la vitesse de convergence vers cette limite.

Si $u$ est de l'ordre de $\frac{\mathrm{I}}{2 \mathrm{~N}}$ nous obtenons :

$$
\begin{aligned}
f(\infty) & =\frac{\mathrm{I}}{\mathrm{I}+4 \mathrm{~N} u}(\mathrm{I}+o(\mathrm{I})) \\
\operatorname{Var}(\mathrm{X}(\infty)) & =\frac{2 \mathrm{~N} u}{3} \frac{(5+2 \mathrm{~N} u)}{(3+4 \mathrm{~N} u)(\mathrm{I}+2 \mathrm{~N} u)(\mathrm{I}+4 \mathrm{~N} u)^{2}}(\mathrm{I}+o(\mathrm{I}))
\end{aligned}
$$




\section{Conclusion}

Nous constatons que le "coefficient de parenté gamétique " tend en moyenne vers I avec une variance qui tend vers o lorsqu'il n'y a pas de mutation. Ce résultat bien connu indique que la population deviendra génétiquement homogène au bout d'un temps suffisant.

En présence de mutation, le coefficient en moyenne ne tend pas vers 0 , il est notablement inférieur à I si le taux de mutation est de l'ordre de $\frac{\mathrm{I}}{2 \mathrm{~N}}$ avec $\mathrm{N}$ grand $(\operatorname{si} 2 \mathrm{~N} u=\mathrm{I}, f(\infty)=0,333 ;$ si $2 \mathrm{~N} u=5, f(\infty)=0,09 \mathrm{I} ; \mathrm{si} 2 \mathrm{~N} u=\mathrm{I0}, f(\infty)=0,047)$. Sa dispersion autour de sa valeur moyenne est assez faible (si $2 \mathrm{~N} u=1$, Var $(\mathrm{X}(\infty))=0,022 ;$ si $2 \mathrm{~N} u=5, \operatorname{Var}(\mathrm{X}(\infty))=0,00 \mathrm{I} 7$, si $2 \mathrm{~N} u=10, \operatorname{Var}(\mathrm{X}(\infty))$ $=0,0004)$. La population présentera alors une notable persistance d'homozygotes.

\section{Summary}

\section{Kinship coefficient and dispersion in a finite random-mating population, incidence of mutation}

The molecular biology shows the existence of numerous polymorphisms. Each substitution of an amino acid for another constitutes a mutation which produces a new gene, a new allele. This phenomenon is expressed by the notion of "perte de parenté ".

The kinship coefficient $\mathrm{X}(t)$ conditionned by the knowledge of the $n$-th generation, namely the probability for two randomly drawn gametes with reinsertion in the gametic pool of the $(n+\mathrm{I})$ - th generation, to coïncide admits as a priori average the kinship coefficient $f(t)$ of MALECOT. The purpose of this paper is to study the interplay beetwen the mutation and the evolution of the a priori variance.

The kinship coefficient $f(t)$ tends to $\mathrm{I}$ in absence of mutation and to $f(\infty)$ whenever the mutation rate is $u$.

The variance of $\mathrm{X}(t)$ tends to $o$ in absence of mutation and to $\operatorname{var}(\mathrm{X}(\infty))$ whenever the mutation rate $u$ is of order of $\frac{\mathrm{I}}{2 \mathrm{~N}}$. The effect of the mutation is to increase the asymptotic value of the variance and to increase the rate of convergence to the limit.

\section{Références bibliographiques}

JACQUARD A., 1975. Inbreeding: one word = several meanings, Théor. Pop. Biology, 7, 338-363.

LEVIANDIER Th., JACQUARD A., I974. Homogénéisation de l'apparentement dans une population limitée. Ann. Génét., 17, 2, 99-103.

KimURA M. and Crow J. F., 1964. The number of allèles that can be maintained in finite populations, Genetics, 49, 725-738.

Malécot G., I966. Probabilités et hérédité, I.N.E.D. Cahier $\mathrm{n}^{0} 47$, Presses universitaires de France.

MALÉcot G., I972. Génétique des populations naturelles dans le cas d'un locus. II. Étude du coefficient de parenté. Ann. Génét. Sél. anim., 4, 385-409.

MALÉCOT G., I973. Génétique des populations diploïdes naturelles dans le cas d'un seul locus. III Parenté, mutations, migrations. Ann. Génét. Sél. anim., 5, 333-36r.

SERANT D., 1976. Sur les processus stochastiques à temps discret introduits par le crossing-over en génétique mathématique. Thèse, Faculté des Sciences de Lyon. 